\title{
Virus-like Particle
}

National Cancer Institute

\section{Source}

National Cancer Institute. Virus-like Particle. NCI Thesaurus. Code C14324.

Non-infectious particle(s) derived from viral structural proteins. These particles are used as vaccines to stimulate a host immune response against the virus that the particles were derived from. 